\title{
Modeling Mesoscale Turbulence in the Barotropic Double-Gyre Circulation
}

\author{
DARRYL D. HOLM \\ T-Division and CNLS, Los Alamos National Laboratory, Los Alamos, New Mexico \\ BALASUBRAMANYA T. NADiga \\ Los Alamos National Laboratory, Los Alamos, New Mexico
}

(Manuscript received 18 April 2002, in final form 4 April 2003)

\begin{abstract}
This paper presents analytical and numerical results for a class of turbulence closure models called "alpha models," in which Lagrangian averaging and turbulence closure assumptions modify the Eulerian nonlinearity. The alpha models are investigated in the setting of the barotropic, double-gyre circulation in an ocean basin. Two variants of the alpha models for the barotropic vorticity (BV) equation are found to produce the correct four-gyre configuration for the mean barotropic circulation in numerical simulations performed at a resolution 4 times as coarse as that required in a resolved BV model. These are the BV- $\alpha$ model and the BV-Leray- $\alpha$ model. However, at a resolution 8 times as coarse, only the BV- $\alpha$ model produces the proper four-gyre configuration. Thus, the combination of modified nonlinearity and viscous dissipation (the viscosity is the same in all of the runs) in the BV- $\alpha$ model is found to provide a promising approach to modeling the mean effects of unresolved mesoscale (subgrid scale) activity in this problem.
\end{abstract}

\section{Introduction}

Studies of wind-driven circulation in a rectangular ocean basin using idealized single- and double-gyre wind forcing have played an important role in understanding various aspects of upper-ocean dynamics, including the role of mesoscale eddies and their effect on mean circulation, as discussed in for example, Holland and Lin (1975) and Holland and Rhines (1980). For example, in the 1970s, mean circulation that was driven entirely by mesoscale eddies was shown to exist in the unventilated subsurface ocean in Holland and Lin (1975).

More recent, in Greatbatch and Nadiga (2000), eddydriven mean flow was shown to emerge in a homogeneous (single layer) model of wind-driven circulation. When the barotropic vorticity (BV) equation is forced by the Ekman pumping that results from a double-gyre wind stress and the dissipation is weak, the instantaneous flow is highly variable, but the time-mean flow shows a distinct four-gyre structure. Two characteristics of this time-mean four-gyre circulation (Greatbatch and Nadiga 2000) are that

1) the two inner gyres circulate in the same direction as conventional wind-driven gyres, while the two

Corresponding author address: B. T. Nadiga, LANL, MS-B296, Los Alamos, NM 87545.

E-mail: balu@lanl.gov outer gyres at the northern and southern ends of the basin circulate in the opposite direction and are driven by the eddy flux of potential vorticity, and

2) the dominant balance is between the wind forcing and the (divergence of the) eddy flux of potential vorticity (with explicit dissipation playing only a minor role).

The eddy flux of potential vorticity that produces the four-gyre structure is fundamentally a transport mechanism that tends to homogenize potential vorticity. In the present case it also gives rise to a four-gyre mean circulation pattern. We emphasize that the formation of the outer gyres is not a linear wave effect, for example, due to Rossby waves. Rather, it is the result of establishing a mean balance primarily between the eddy flux of potential vorticity and wind forcing. When the relative importance of the eddy flux of potential vorticity is reduced by increasing the explicit dissipation, the outer counterrotating gyres disappear and only a conventional double-gyre circulation appears in the time mean (see Fig. 8 of Greatbatch and Nadiga 2000). The emergence of such a four-gyre pattern in the time mean circulation from a double-gyre wind forcing is quite robust and does not depend sensitively on the Rossby number, nor does it depend on the form of dissipation or the boundary conditions, as long as the dissipation is sufficiently weak. However, this distinctive four-gyre mean circulation pattern generated by eddy transport is susceptible to destruction by excessive dissipation. 
The effects of mesoscale eddies on mean circulation are related to the exchange between the mean and eddy components of both kinetic and potential energies, and also to the mixing of tracers. In the present context of the barotropic vorticity equation, only the first of these three interactions exists, and so our considerations will be limited to aspects related to effects on transport by parameterizations of horizontal Reynolds stresses. Previous parameterizations of horizontal Reynolds stresses [either in simple models, or in ocean general circulation models (OGCMs)] have been largely devoted to downgradient mixing, either of momentum or of potential vorticity (e.g., see Peterson and Greatbatch 2001). Other approaches include those based on statistical mechanics (e.g., see Holloway 1986) and the method of "anticipated potential vorticity" of Sadourny and Basdevant (1985), which conserves energy while dissipating potential enstrophy in the quasigeostrophic approximation. All of these previous parameterizations are fundamentally dissipative.

These dissipative parameterizations of mesoscale eddy transport may be beneficial from the viewpoint of numerical stability. In fact, underresolved numerical simulations in oceanography are often stabilized by artificially increasing viscosity. However, increasing viscosity tends to diminish variability. Alternatively, one may modify the nonlinearity to produce the desired numerical stability at a lower viscosity. The latter approach tends to preserve the variability seen in the higher-resolution (lower viscosity) runs. However, in modifying the nonlinearity, one must take care to preserve fundamental circulation properties such as Kelvin's theorem and local conservation of potential vorticity (PV). Otherwise, one may not properly capture the desired physical phenomena (the four-gyre mean circulation effect in this case).

Recently, a new type of turbulence closure called Lagrangian-averaged Navier-Stokes alpha (LANS- $\alpha$ ) equations have been proposed and tested for incompressible three-dimensional turbulent flows. The alpha terms in these models alter the fluid nonlinearity without introducing additional dissipative terms beyond those in the Navier-Stokes equations. Instead, in these "alpha models," the nonlinearity is altered so that excitations at length scales that are smaller than the given length scale alpha $(\alpha)$ are nonlinearly swept by motions at the larger length scales. Moreover, these smaller excitations do not interact among themselves to cascade into still smaller ones. (In numerics, the natural choice for the length scale alpha is the resolution scale.) The main effect of the alpha terms is to make the LANS- $\alpha$ solution smoother than it would be in their absence, but we emphasize this is accomplished without introducing additional dissipative terms or augmenting the value of the viscosity coefficient. Instead, the nonlinear transport terms are modified in the alpha-model equations. Explicitly, the transport velocity is filtered relative to the transported velocity as done in Leray (1934) for the
Navier-Stokes equations. Moreover, an additional nonlinear alpha term beyond the Leray component of the alpha model is included to restore the Kelvin circulation theorem. In the context of the BV equation, this additional nonlinear alpha term restores the potential vorticity (PV) advection form of the inviscid BV- $\alpha$ model [Eq. (10) of Nadiga and Margolin (2001)].

Preliminary investigations of two-dimensional alpha models were considered in Nadiga (2000) and Nadiga and Shkoller (2001), and a test of these 2D alpha models in an application to the double-gyre circulation was given in Nadiga and Margolin (2001). The latter paper offered a first step in assessing the utility of the alphamodel approach for simulating complex 2D geophysical flows, by showing its ability to qualitatively reproduce the time-mean four-gyre structure. In that work, the approach taken was to model the Reynolds stresses to recover just the time-mean circulation (as a steady-state solution of the modeled equation) and the choice of boundary conditions was found to be an important aspect of the modeling. The present work follows the large-eddy-simulation (LES) approach and thus addresses the issue of modeling the effects of turbulence transport by unresolved eddies as well. Consequently, the philosophy is different also: Given an eddy-resolving simulation, we attempt to reproduce its time-mean circulation and a few of its important turbulence correlations at a much coarser (non-eddy-resolving) resolution. While we attempt this with different models, we shall hold all other parameterizations fixed. In particular, we will always set the filter scale alpha equal to the a priori specified coarse resolution and hold the coefficient of viscosity fixed while changing resolution. Thus, we model the effects of eddy transport in the nonlinear transport term itself, instead of modeling it as an eddy diffusivity, as is often done in traditional approaches. Eddy-driven transport tends to homogenize potential vorticity. The LANS- $\alpha$ model performs a similar role by causing the excitations at length scales smaller than alpha to be swept passively by the fluid motions at larger length scales.

Following the procedure introduced for the numerical study of the turbulent mixing layer in Geurts and Holm (2002), the present work splits the alpha model corresponding to the barotropic vorticity equation (BV $\alpha)$ into several LES-like mean stress components and considers their separate effects on the preservation of the eddy-driven (time mean) four-gyre circulation in the double-gyre problem. Such a decomposition first places the BV- $\alpha$ model into the familiar LES framework and then distinguishes the $\mathrm{BV}-\alpha$ model and its various alpha terms from previous models of eddy transport in the geophysical setting. The models are compared using the standard viscous (Laplacian) dissipation of relative vorticity in conjunction with slip boundary conditions (homogeneous Dirichlet boundary conditions on the vorticity).

We note that in using the LES philosophy, we will 
not claim to model what is traditionally regarded as a turbulent flow (with its wide range of spatial scales and an inertial subrange). Rather, we will first establish that nonlinear (eddy) transport is the physical mechanism responsible for the four-gyre pattern in the mean circulation and then compare the qualitative changes in our numerical simulations using the standard LES philosophy_coarse resolution and no model, versus coarse resolution and two different alpha models, all compared with high-resolution numerics as the reference. Thus, we shall adopt the LES approach to model mean eddy transport in the highly variable (but not, perhaps, classically turbulent) double-gyre problem. Rather than replacing the transport effects of unresolved eddies by viscous diffusion, our approach modifies the fluid transport nonlinearity to model the transport effects of unresolved eddies.

In section 2 we review the LANS- $\alpha$ equations and their properties such as momentum conservation, the Kelvin circulation theorem, and potential-vorticity conservation. We also review the relation of the LANS- $\alpha$ model to the historical model of Leray (1934) and the LES models of turbulence based on Leonard (1973) and Clark et al. (1979). We then reformulate the LANS- $\alpha$ equations using a commutator identity to facilitate the reduction of the LANS- $\alpha$ model to its LES-like submodels. In section $2 \mathrm{e}$ we specialize to two dimensions and discuss the models that we shall compare for the double-gyre simulations. Then in section 3 we describe the main results for the $\mathrm{BV}-\alpha$ simulations and we compare these results with the corresponding high-resolution results and other model results at resolutions 4 and 8 times as coarse. Section 4 presents our conclusions and outlook for future investigations.

\section{Lagrangian-averaged Navier-Stokes- $\alpha$ equation}

In the large-eddy simulation of a turbulent flow, one attempts to model the dynamics of the smaller resolved scales so as 1) to reproduce the energy transfer characteristics (between the large resolved scales and the small unresolved scales) that would occur if the turbulent flow were fully resolved and 2) to dissipate the correct amount of energy. The LES approach can be viewed as a turbulence closure that specifies an additional stress-strain relationship modeling the effects of the unresolved scales on the numerically resolved scales in terms of the resolved flow characteristics. Thus, although the constitutive nature of the fluid is known at the molecular scale, the LES closure of the equations governing the large scales is formally equivalent to specifying a new constitutive law for the stress tensor at the larger, resolved, scales of the flow. This effective constitutive law for the resolved scales is imposed by the choices of the Eulerian averaging/filtering process and the closure assumptions.

The Lagrangian-averaged Navier-Stokes $\alpha$ (LANS$\alpha$ ) model describes the Lagrangian mean motion of an incompressible fluid whose equations are closed by assuming the turbulence at length scales below alpha is "frozen" into the Lagrangian mean flow according to the hypothesis of Taylor (1938). As a result, the alpha model regularizes the Navier-Stokes (NS) equations by modifying their nonlinearity so that scales smaller than alpha are swept by the larger scales, while preserving their fundamental inviscid transport properties such as convection of vorticity. The properties of the NavierStokes- $\alpha$ equations, their numerical performance, and the comparisons of their solutions with classical turbulence experiments are reviewed in Chen et al. (1998, 1999), Marsden and Shkoller (2001), Foias et al. (2001, 2002), and references therein.

The LANS- $\alpha$ equations for incompressible flow in a rotating frame are given by

$$
\begin{aligned}
\partial_{t} \mathbf{v} & +\underset{\text { modified nonlinearity }}{\mathbf{u}_{\mathbf{u}} \cdot \boldsymbol{\nabla \mathbf { v }}+\boldsymbol{\nabla} \mathbf{u}^{\mathrm{T}} \cdot \mathbf{v}} \\
& +\boldsymbol{\nabla}\left(p-\frac{1}{2}|\mathbf{u}|^{2}-\frac{\alpha^{2}}{2}|\nabla \mathbf{u}|^{2}\right)-\mathbf{u} \times \mathbf{f}=\nu \Delta \mathbf{v}+\mathbf{F}
\end{aligned}
$$

in which two velocities appear in the term labeled "modified nonlinearity." Here $\mathbf{v} \equiv \mathbf{u}-\alpha^{2} \Delta \mathbf{u}$ is the specific momentum associated with the fluid velocity $\mathbf{u}$, which is incompressible $(\boldsymbol{\nabla} \cdot \mathbf{u}=0)$. The above system is completed by prescribing the usual boundary condition of $\left.\mathbf{u} \cdot \hat{\mathbf{n}}\right|_{\partial \Omega}=0$ (no normal flow) at a fixed wall, and another condition on the tangential velocity at the wall, $\left.\mathbf{u} \cdot \hat{\mathbf{t}}\right|_{\partial \Omega}=0$ (no slip), for example, or $\boldsymbol{\nabla}(\mathbf{u} \cdot \hat{\mathbf{t}})$. $\left.\hat{\mathbf{n}}\right|_{\partial \Omega}=0$ (slip). These boundary conditions are sufficient to perform the inversion of the Helmholtz operator, required for determining the $\mathbf{u}$ velocity from the $\mathbf{v}$ velocity. In other notation, $\nabla \mathbf{u}^{\mathrm{T}} \cdot \mathbf{v}=\sum_{j=1}^{3} v_{j} \nabla u^{j}, p$ is pressure, $\mathbf{f}=f \hat{\mathbf{z}}$ is the constant Coriolis vector, $\nu$ is the constant kinematic viscosity, and $\mathbf{F}$ is an external force.

We shall explain the sum of two terms labeled modified nonlinearity in (1) by considering its Kelvin circulation theorem as follows.

\section{a. Modified nonlinearity and Kelvin's circulation theorem}

Equation (1) satisfies the modified Kelvin's circulation theorem,

$$
\frac{d}{d t} \oint_{c(\mathbf{u})}\left(\mathbf{v}+f \hat{\mathbf{z}} \times \frac{\mathbf{x}}{2}\right) d \mathbf{x}=\oint_{c(\mathbf{u})}(\nu \Delta \mathbf{v}+\mathbf{F}) d \mathbf{x},
$$

for a fluid loop $c(\mathbf{u})$ that moves with Helmholtz-filtered velocity $\mathbf{u}=\left(1-\alpha^{2} \Delta\right)^{-1} \mathbf{v}$, interpreted in Chen et al. (1998) as the Lagrangian-mean fluid velocity. This circulation theorem is verified directly by taking the time derivative inside the integral around the Kelvin loop $c(\mathbf{u})$ in (2) to find 


$$
\begin{aligned}
& \frac{d}{d t} \oint_{c(\mathbf{u})}\left(\mathbf{v}+f \hat{\mathbf{z}} \times \frac{\mathbf{x}}{2}\right) d \mathbf{x} \\
& \quad=\oint_{c(\mathbf{u})}(\partial_{t} \mathbf{v}+\underbrace{\mathbf{u} \cdot \nabla \mathbf{v}+\nabla \mathbf{u}^{\mathrm{T}} \cdot \mathbf{v}}_{\text {modified nonlinearity }}-\mathbf{u} \times f \hat{\mathbf{z}}) d \mathbf{x} .
\end{aligned}
$$

The modified Kelvin circulation theorem (2) then follows upon substituting the motion equation (1) into identity (3) and using $\oint_{c(\mathbf{u})} \boldsymbol{\nabla} \pi \cdot d \mathbf{x}=0$ for any continuous function $\pi$. Thus, the modified nonlinearity in the motion equation (1) for the NS- $\alpha$ model provides the Kelvin circulation theorem (2), in which the fluid loop moves with Helmholtz-filtered velocity $\mathbf{u}=(1-$ $\left.\alpha^{2} \Delta\right)^{-1} \mathbf{v}$ instead of the corresponding "defiltered" velocity v. (As specified earlier, the boundary conditions on $\mathbf{u}$ are sufficient to invert the Helmholtz operator in determining the $\mathbf{u}$ velocity from the $\mathbf{v}$ velocity.) This circulation theorem is the result of Lagrangian averaging in combination with the particular Taylor-hypothesis closure assumption discussed, for example, in Foias et al. (2001). For more details of this closure, see Holm (1999). In the present context, the circulation theorem (2) provides an interpretation of the two velocities in the modified nonlinearity in the LANS- $\alpha$ motion equation (1). The fluid parcels that compose the Kelvin loop move with the filtered transport velocity $\mathbf{u}$. The other velocity $\mathbf{v}$ in the integrand of the circulation theorem (2) is the defiltered velocity associated with the mean momentum. The mean momentum appears in Newton's law for the mean evolution of the fluid, which is expressed in the NS- $\alpha$ motion equation.

\section{b. Comparing LANS- $\alpha$ model with Leray regularization of Navier-Stokes}

Combining incompressibility $(\boldsymbol{\nabla} \cdot \mathbf{u}=0)$ and the vector calculus identity,

$$
\begin{gathered}
\boldsymbol{\nabla} \mathbf{u}^{\mathrm{T}} \cdot \mathbf{v}-\boldsymbol{\nabla}\left(\frac{1}{2}|\mathbf{u}|^{2}+\frac{\alpha^{2}}{2}|\nabla \mathbf{u}|^{2}\right) \\
=-\alpha^{2} \operatorname{div}\left(\nabla \mathbf{u}^{\mathrm{T}} \cdot \nabla \mathbf{u}\right),
\end{gathered}
$$

allows the LANS- $\alpha$ motion equation (1) to be rewritten equivalently in index notation (summing on repeated indices) as a momentum balance relation:

$$
\partial_{t} v_{i}+\partial_{k} T_{i}^{k}-\epsilon_{i j k} u^{j} f^{k}=\nu \Delta v_{i}+F_{i} .
$$

This expression of LANS- $\alpha$ motion introduces the stress tensor

$$
T_{i}^{k}=v_{i} u^{k}+p \delta_{i}^{k}-\underbrace{\alpha^{2} u_{j, i} u^{j, k}}_{\begin{array}{c}
\text { drop for } \\
\text { Leray- } \alpha \text { model }
\end{array}} .
$$

Thus, the modification of the nonlinearity in the LANS$\alpha$ motion equation (1) translates into a modification of the mean stress tensor in (6), whose last term is the right-hand side of the vector identity (4). The Leray- $\alpha$ model appears when the last term of this modified stress tensor is dropped. Dropping the last term in (6) corresponds to dropping both the term $\nabla \mathbf{u}^{\mathrm{T}} \cdot \mathbf{v}$ and the pressure modifications in the LANS- $\alpha$ motion equation (1). That is, dropping this term relinquishes the modified Kelvin circulation theorem (2), and gives a variant of the Leray regularization of the Navier-Stokes equations (Leray 1934). In this variant, the Leray-filtering of the components of $\mathbf{v}$ to produce the smoothed transport velocity $\mathbf{u}=g \times \mathbf{v}$ is accomplished by inverting the Helmholtz operator in the relation $\left(1-\alpha^{2} \Delta\right) \mathbf{u}=\mathbf{v}$ using the homogeneous Dirichlet boundary condition $\left.\mathbf{u}\right|_{\partial \Omega}=$ 0 . This variant of the Leray model (called the Leray- $\alpha$ model in Geurts and Holm 2002) is given by

$$
\partial_{t} \mathbf{v}+\mathbf{u} \cdot \nabla \mathbf{v}+\nabla p-\mathbf{u} \times \mathbf{f}=\nu \Delta \mathbf{v}+\mathbf{F},
$$

where

$\mathbf{v} \equiv \mathbf{u}-\alpha^{2} \Delta \mathbf{u}, \quad \boldsymbol{\nabla} \cdot \mathbf{u}=0, \quad$ and $\left.\quad \mathbf{u}\right|_{\partial \Omega}=0$.

One may consult Geurts and Holm (2002) for discussions of the performance of the Leray- $\alpha$ model in numerically simulating the dynamics of a turbulent mixing layer in three dimensions.

\section{c. Comparing LANS- $\alpha$ with LES fluid models}

The commutator of the advection operator $d / d t \equiv \partial_{t}$ $+\mathbf{u} \cdot \boldsymbol{\nabla}$ and the Helmholtz operator $\left(1-\alpha^{2} \Delta\right)$ acting on the fluid velocity $\mathbf{u}$ satisfies the remarkable identity,

$$
\begin{aligned}
{\left[\frac{d}{d t},\left(1-\alpha^{2} \Delta\right)\right] \mathbf{u}=} & \alpha^{2} \operatorname{div}\left(\nabla \mathbf{u} \cdot \nabla \mathbf{u}+\nabla \mathbf{u} \cdot \nabla \mathbf{u}^{\mathrm{T}}\right) \\
& -\alpha^{2}[\nabla(\operatorname{div} \mathbf{u}) \cdot \nabla] \mathbf{u} .
\end{aligned}
$$

For incompressible flow, div $\mathbf{u}=0$, so the last term vanishes in this commutator relation. Substituting the commutator identity (8) into motion equation (1) and again using the vector identity (4) for $\nabla \mathbf{u}^{\mathrm{T}} \cdot \mathbf{v}=\sum_{j=1}^{3}$ $v_{j} \nabla u^{j}$ allows the modified nonlinearity in the LANS- $\alpha$ equation (1) to be rewritten equivalently as

$$
\begin{gathered}
\left(1-\alpha^{2} \Delta\right)\left(\partial_{t} \mathbf{u}+\mathbf{u} \cdot \nabla \mathbf{u}-\nu \Delta \mathbf{u}\right)+\nabla p \\
-\mathbf{u} \times \mathbf{f}-\mathbf{F}+\alpha^{2} \operatorname{div} \tau=0,
\end{gathered}
$$

where the stress divergence is given by

$$
\operatorname{div} \tau \equiv \operatorname{div}(\underbrace{\nabla \mathbf{u} \cdot \nabla \mathbf{u}^{\mathrm{T}}}_{\text {Clark stress }}+\underbrace{\nabla \mathbf{u} \cdot \boldsymbol{\nabla u}-\nabla \mathbf{u}^{\mathrm{T}} \cdot \boldsymbol{\nabla u}}_{\text {other LANS- } \alpha \text { stresses }}),
$$

or, in components,

$$
(\operatorname{div} \tau)_{i}=\partial_{k}\left(u_{i, j} u^{k, j}+u_{i, j} u^{j, k}-u_{j, i} u^{j, k}\right) .
$$

Finally, the pressure $p$ is determined by solving the Neumann problem obtained from preservation of incompressibility, div $\mathbf{u}=0$, subject to the boundary conditions specified for $\mathbf{u}$ and the normal component of the 
motion equation (9) vanishing on the boundary. This is how the stress tensor $\tau$ in (9) affects the solution for the pressure $p$ in incompressible flow.

One of the three summands in the nonsymmetric tensor $\tau$ in (10) or (11) is already familiar from LES modeling: the symmetric tensor with components $\left(\boldsymbol{\nabla u} \cdot \nabla \mathbf{u}^{\mathrm{T}}\right)_{i}^{k}=u_{t, j} u^{k, j}$ is known in LES modeling as the Clark, or Leonard stress, for the "tensor diffusivity model" obtained by filtering the NS equations (Leonard 1973). In particular, $\operatorname{div}\left(\boldsymbol{\nabla u} \cdot \nabla \mathbf{u}^{\mathrm{T}}\right)=u_{i, j k} S^{j k}$, and so the strain-rate tensor $S^{j k}=(1 / 2)\left(u^{k, j}+u^{j, k}\right)$ "plays the role of a tensorial effective viscosity" whose antidiffusive aspects are discussed in Winckelmans et al. (2001) and references therein. In contrast, the other LANS- $\alpha$ stress tensors in (11) do not arise in applying Eulerian filtering to the NS equations and, to our knowledge, they had not been used in modeling turbulence before the LANS$\alpha$ model was developed. As mentioned earlier, dropping the last term in the stress tensor (10) recovers the variant of the Leray regularization that uses inversion of the Helmholtz operator as its filter in smoothing the transport velocity.

The rest of this paper explores the effects of using Helmholtz filtering in the 2D quasigeostrophic doublegyre situation. This is done by comparing the mean dynamical behavior of the eddy-resolving simulations for the original equations with the corresponding behavior using the appropriate LANS- $\alpha$ equations (1) and the Leray- $\alpha$ equations (7) at coarser resolution. In this 2D quasigeostrophic situation, these equations simplify considerably.

\section{d. $2 D$ reductions of the $L A N S-\alpha$ vorticity equation}

By using the standard vector identity for fluid dynamics,

$$
\mathbf{u} \cdot \boldsymbol{\nabla} \mathbf{v}+\nabla \mathbf{u}^{\mathrm{T}} \cdot \mathbf{v}=-\mathbf{u} \times \operatorname{curl} \mathbf{v}+\boldsymbol{\nabla}(\mathbf{u} \cdot \mathbf{v}),
$$

one may rewrite the LANS- $\alpha$ equations in (1) as

$$
\begin{aligned}
\partial_{t} \mathbf{v} & -\mathbf{u} \times(\operatorname{curl} \mathbf{v}+\mathbf{f}) \\
& +\nabla\left(\mathbf{u} \cdot \mathbf{v}+p-\frac{1}{2}|\mathbf{u}|^{2}-\frac{\alpha^{2}}{2}|\nabla \mathbf{u}|^{2}\right)=\nu \Delta \mathbf{v}+\mathbf{F}
\end{aligned}
$$

where

$$
\boldsymbol{\nabla} \cdot \mathbf{u}=0 \text { and }\left.\mathbf{u}\right|_{\partial \Omega}=0 .
$$

Hence, for constant $\alpha$ and incompressible flow, $\boldsymbol{\nabla} \cdot \mathbf{u}=$ 0 , taking the curl of the LANS- $\alpha$ equations (1) implies the $3 \mathrm{D}$ vorticity equation for $\mathbf{q}=$ curlv,

$$
\begin{aligned}
\partial_{t} \mathbf{q} & +\mathbf{u} \cdot \nabla(\mathbf{q}+\mathbf{f})-(\mathbf{q}+\mathbf{f}) \cdot \nabla \mathbf{u} \\
= & \nu \Delta \mathbf{q}+\operatorname{curl} \mathbf{F} .
\end{aligned}
$$

When $\alpha^{2} \rightarrow 0$, (13) reduces to Helmholtz vorticity dynamics for the Navier-Stokes equations in a nonuniformly rotating frame.

In two planar dimensions, the LANS- $\alpha$ vorticity equa- tion (13) reduces to evolution of the scalar potential vorticity, $q+\beta y=\hat{\mathbf{z}} \cdot \operatorname{curl} \mathbf{v}+\beta y$, which is conserved in the inviscid unforced case. That is, after taking $\mathbf{f}=$ $\hat{\mathbf{z}} f$ with $f=f_{0}+\beta y$ in two planar dimensions $(x, y)$ with constants $f_{0}$ and $\beta$, (13) reduces to the $\mathrm{BV}-\alpha$ equation in 2D:

$$
\partial_{t} q+\mathbf{u} \cdot \nabla(q+\beta y)=\nu \Delta q+\hat{\mathbf{z}} \cdot \operatorname{curl} \mathbf{F} .
$$

As compared with (13), this BV- $\alpha$ equation has no vortex-stretching term.

We note that the BV- $\alpha$ model (14) can alternatively be derived by first applying the Taylor hypothesis for the alpha closure in the shallow-water approximation, and then moving into a rotating frame, assuming rapid rotation, performing asymptotic expansion in Rossby number, and retaining only leading-order dynamics. This ordering of operations in the derivation of BV- $\alpha$ model is valid because the motion equation for the alpha closure in the shallow-water approximation transforms correctly under moving into a rotating frame. Furthermore, since the alpha model retains the Helmholtz form for its vorticity dynamics, Rossby plane waves are still nonlinear solutions of the BV- $\alpha$ model, although their linear dispersion relation is modified by including alpha effects:

$$
\omega=U k-\frac{\beta k}{|\mathbf{k}|^{2}\left(1+|\mathbf{k}|^{2} \alpha^{2}\right)},
$$

where $U$ is the ambient zonal flow, $|\mathbf{k}|^{2}=k^{2}+l^{2}, k$ is the wavenumber in the east-west direction, and $l$ is the wavenumber in the north-south direction. Rossby waves whose wavenumber $|\mathbf{k}|$ satisfies $|\mathbf{k}| \alpha \ll 1$ are essentially unmodified in the BV- $\alpha$ model. However, Rossby waves whose wavenumber $|\mathbf{k}|$ satisfies $|\mathbf{k}| \alpha$ $\gg 1$ do not propagate at all in the $\mathrm{BV}-\alpha$ model; that is, phase speed relative to the ambient flow goes to zero as $|\mathbf{k}|^{-4}$.

The corresponding BV equation for the Leray- $\alpha$ model in two planar dimensions is the BV-Leray- $\alpha$ equation,

$$
\partial_{t} q+\mathbf{u} \cdot \nabla(q+\beta y)+\left\{u_{j}, v^{j}\right\}=\nu \Delta q+\hat{\mathbf{z}} \cdot \operatorname{curl} \mathbf{F},
$$

where the difference $\left\{u_{j}, v^{j}\right\}$ between (14) and (16) is expressed in terms of the streamfunction $\psi$ for the filtered velocity $\mathbf{u}=-\hat{\mathbf{z}} \times \boldsymbol{\nabla} \psi$ as

$$
\begin{aligned}
& \left\{u_{j}, v^{j}\right\} \equiv \hat{\mathbf{z}} \cdot \nabla u_{j} \times \nabla v^{j} \\
& \quad=\left(\psi_{x x}-\psi_{y y}\right) H_{\alpha} \psi_{x y}-\psi_{x y} H_{\alpha}\left(\psi_{x x}-\psi_{y y}\right) .
\end{aligned}
$$

Here $H_{\alpha}=1-\alpha^{2} \Delta$ is the Helmholtz operator with constant length scale $\alpha$. We sum over repeated indices and $\{a, b\}=a_{x} b_{y}-b_{x} a_{y}$ is the Jacobi bracket. Note that the terms dropped from the LANS- $\alpha$ motion equation (1) in obtaining the Leray-alpha model (7) now lead to additional terms (17) in the BV-Leray- $\alpha$ equation (16), since PV is not conserved for the Leray-alpha model. This is the effect of having relinquished the Kelvin circulation theorem in dropping those terms. 

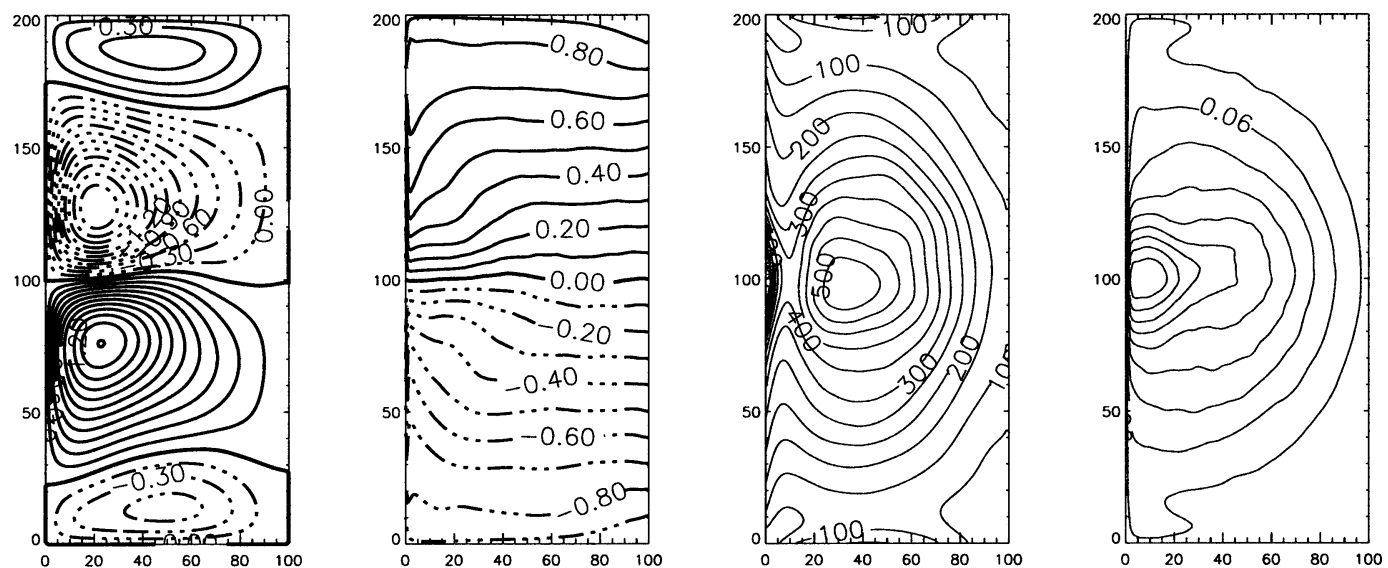

FIG. 1. Contour plots of time-averaged fields from the high-resolution simulation. From left to right: streamfunction, potential vorticity (PV), eddy kinetic energy (EKE), and eddy potential enstrophy (EPE). The Munk layer scale is $0.02 \mathrm{~L}$ and the grid resolution is $0.01 \mathrm{~L}$. At this low level of viscosity, the time-mean streamfunction displays a fourgyre structure even though the wind forcing is that for a double gyre.

\section{e. Three variants: $B V, B V$ - $\alpha$, and $B V$-Leray- $\alpha$}

We shall consider the following three variants of the $\mathrm{BV}$ equations in which $\omega=\Delta \psi$ is the relative vorticity and $H_{\alpha} \omega=q$, with $\left.\omega\right|_{\partial \Omega}=0$. This boundary condition on $\omega$ is consistent with the slip boundary condition on u. The first of these three variants represents the usual BV equation rewritten in terms of the velocity $\mathbf{v}$ and acted on by Helmholtz-operator inversion to obtain an evolution equation for vorticity $\omega$. The second variant is (14) and the third is (16). Again, each equation is acted on by Helmholtz-operator inversion to obtain an equation for vorticity $\omega=H_{\alpha}^{-1} q$. We rewrite these equations in this parallel formulation for $\omega$ evolution in $2 \mathrm{D}$, so that they may be conveniently compared on the same basis in the numerical results that follow.

BV model:

$\partial_{t} \omega+H_{\alpha}^{-1}\left(\mathbf{v} \cdot \nabla q-\beta H_{\alpha} \psi_{x}-\nu \Delta q-\hat{\mathbf{z}} \cdot \operatorname{curl} \mathbf{F}\right)=0$,

BV- $\alpha$ model [cf (14)]:

$$
\partial_{t} \omega+H_{\alpha}^{-1}\left(\mathbf{u} \cdot \nabla q-\beta \psi_{x}-\nu \Delta q-\hat{\mathbf{z}} \cdot \operatorname{curl} \mathbf{F}\right)=0,
$$

BV-Leray- $\alpha$ model [cf. (16)]:

$$
\begin{aligned}
& \partial_{t} \omega+H_{\alpha}^{-1}\left(\mathbf{u} \cdot \nabla q-\beta \psi_{x}-\nu \Delta q-\hat{\mathbf{z}} \cdot \operatorname{curl} \mathbf{F}\right) \\
& \quad+H_{\alpha}^{-1}\left[\left(\psi_{x x}-\psi_{y y}\right) H_{\alpha} \psi_{x y}-\psi_{x y} H_{\alpha}\left(\psi_{x x}-\psi_{y y}\right)\right]=0 .
\end{aligned}
$$

All three models describe planar $\omega$ evolution. Two differences occur between the BV and BV- $\alpha$ models, one in the nonlinear transport and the other in the advection of planetary vorticity. The terms that are dropped for the Leray-alpha model now lead to additional terms in the BV-Leray- $\alpha$ equation, since PV is not conserved for the Leray model. We shall impose the condition $\omega=0$ on the boundary. This boundary condition allows one to invert the Helmholtz operator in obtaining the $\mathbf{u}$ vorticity $\omega$ from the $\mathbf{v}$ vorticity $q=\left(1-\alpha^{2} \Delta\right) \omega$.

A BV- $\alpha$ variant of the Clark, or tensor diffusivity, LES model also exists, which is obtained by dropping the other two LANS- $\alpha$ stresses appearing in the stress divergence (10). We shall comment briefly on the performance of this model in comparison with the others, where appropriate. As might be expected from its antidiffusive properties, however, the Clark BV- $\alpha$ model showed unstable behavior in the double-gyre configuration, even when filtered by the Helmholtz inversion, $H_{\alpha}^{-1}$.

\section{Results of the double-gyre simulations}

As discussed in the introduction, when the barotropic vorticity equation (18) is forced by the Ekman pumping resulting from a double-gyre wind stress $\left[F=F_{0}\right.$ $\sin (\pi y)]$ and the dissipation is weak, the instantaneous flow is highly variable (not to say turbulent), and the time-mean flow shows a four-gyre structure. Figure 1 shows four panels containing level contours of, respectively, the time-mean streamfunction (contour increment $=0.15)$, potential vorticity (contour increment $=0.10$ ), eddy kinetic energy (EKE; contour increment $=50$ ), and eddy potential enstrophy (EPE; contour increment $=0.03$ ) for such a case. For future reference, all of the remaining figures will be presented in the same format and the contour increments will be held the same. The Rossby number, defined as $U / \beta L^{2}$ (where the reference velocity $U$ is the Sverdrup velocity, and the reference length $L$ is the horizontal extent of the domain), in this case was 0.0016 , the Munk layer scale was $0.02 L$ and slip boundary conditions were used. Unless stated otherwise, each of these settings will be identical in all the runs to be presented. The flow, for which the time-mean circulation is shown in Fig. 1, was resolved on a 101 

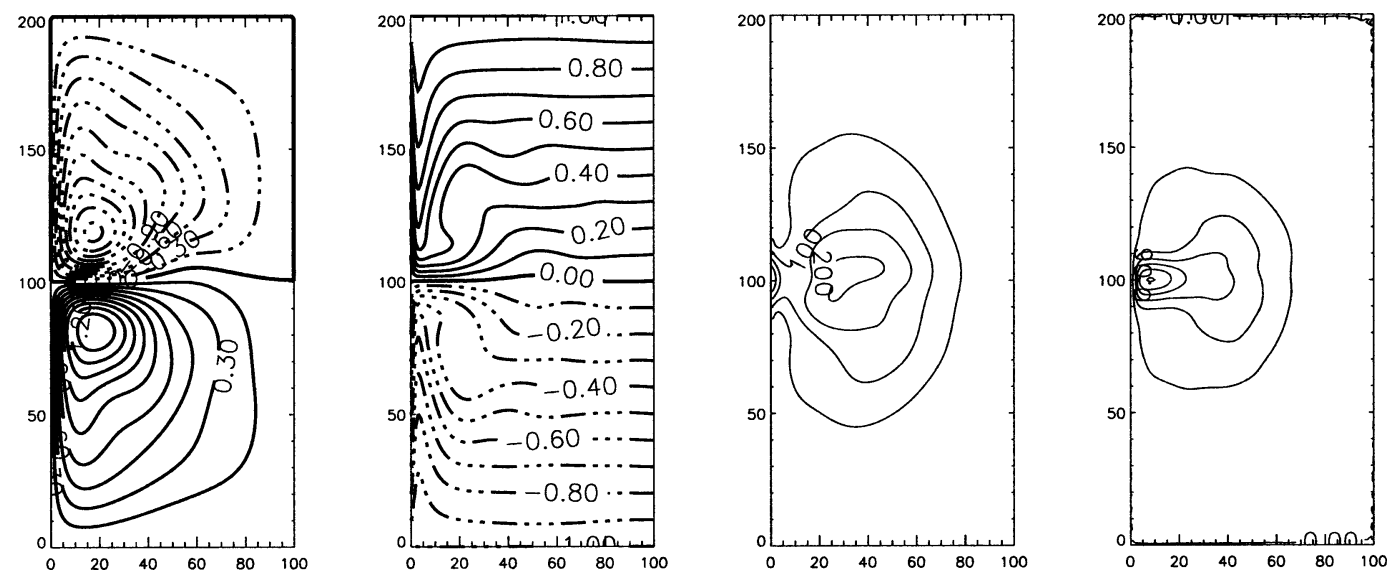

FIG. 2. At a higher viscosity (corresponding to a Munk layer scale of $0.03 \mathrm{~L}$ ), the eddy flux of PV is so reduced that the outer pair of inertial gyres disappears and a classic double-gyre configuration emerges in the time mean. The format of this figure is the same as in Fig. 1.

$\times 201$ grid giving a grid spacing $\Delta x$ of $0.01 L$, which is enough to resolve the Munk scale, and alpha was set to zero. Unless stated otherwise, all computations presented in this paper were carried out using second-order finite-difference spatial discretization with the Arakawa Jacobian, which conserves both kinetic energy and potential enstrophy and a (nominally) fifth-order, adaptive time step, embedded Runge-Kutta Cash-Karp algorithm for the time-marching scheme. (For details, see Greatbatch and Nadiga 2000.)

For the resolved case with weak viscous dissipation shown in Fig. 1, an outer pair of eddy-driven gyres is present, in addition to the usual inner pair of winddriven gyres. The outer eddy-driven gyres are absent, however, in Fig. 2. In the run for which the time-mean circulation is shown in Fig. 2, an elevated viscosity coefficient was used, corresponding to a Munk layer scale of $0.03 L$.

The velocity used in the definition of the Rossby number is the Sverdrup velocity-the velocity that would result in the gyre interior through linear dynamics; Rossby number thus defined is a convenient way of specifying the forcing strength. So, the actual inertial width of the WBC in the time-mean circulation will depend on the extent of the turbulence itself: though the specified Rossby number is the same is Figs. 1 and 2, since mesoscale turbulence in Fig. 1 is stronger than in Fig. 2, the width of the WBC in Fig. 1 is much more than in Fig. 2.

In this context, we also note that the usual estimate of the inertial width of the WBC as $\sqrt{U / \beta}$ is based on the laminar, although highly inertial flow hypothesis that the streamlines are isolines of potential vorticity, or that particles do not cross potential vorticity contours. This is clearly not the case presently. The peak value of the time-mean streamfunction in the inner wind-driven gyres in Fig. 1 is about 1.5, so that the above estimate for the inertial width of the WBC would be about $0.05 \mathrm{~L}$ ( $\sqrt{1.5 R o})$. However, the width is seen in Fig. 1 to be more like $0.25 \mathrm{~L}$, scaling better with the width estimated using the turbulent velocity resulting in the actual simulation: From the contour plot of eddy kinetic energy in Fig. 1, the peak turbulent velocity is about 30, so that the estimate for the width of the turbulent WBC is about $0.22 L$. Thus the present situation is not unlike the much more rapid growth of the turbulent boundary layer thickness $\left(\propto x^{6 / 7}\right)$ with downstream distance $x$ from the leading edge than that of the laminar (Blasius) boundary layer thickness $\left(\propto x^{1 / 2}\right)$ on a flat plate.

We also wish to remind the reader that, in effect, two different averaging procedures are being used in the discussion of the simulations. The first of these is the spatial averaging or filtering procedure, denoted by $H_{\alpha}^{-1}$ in the governing equations previously discussed, and which arises as the inverse of the Helmholtz operator introduced by the alpha-modeling approach. Through the turbulence closure procedure, this Lagrangian averaging results in spatial filtering, specified as inversion of the Helmholtz operator. The boundary condition, $\left.\omega\right|_{\partial \Omega}=0$ allows this Helmholtz operator inversion to be performed uniquely. After this Lagrangian averaging procedure and closure that generates the alpha models, we perform the usual temporal averaging at fixed Eulerian position, for which issues of boundary conditions do not arise. In general, these two averaging procedures do not commute.

Figure 3 shows that the BV simulation loses the fourgyre configuration when its resolution is coarsened by a factor of 4 in each direction. Clearly, at the 4-timesas-coarse resolution some additional modeling would be required to reacquire the four-gyre configuration. Furthermore, the grid resolution of $0.04 \mathrm{~L}$ is too coarse to resolve the viscous boundary layer (Munk scale of $0.02 L)$. This shows up as the grid-scale oscillation in the time-averaged potential-vorticity contours of Fig. 3. Note, however, that the resolution of $0.04 \mathrm{~L}$ is sufficient to resolve the width of the turbulent WBC $(\approx 0.25 L)$ in Fig. 1. 

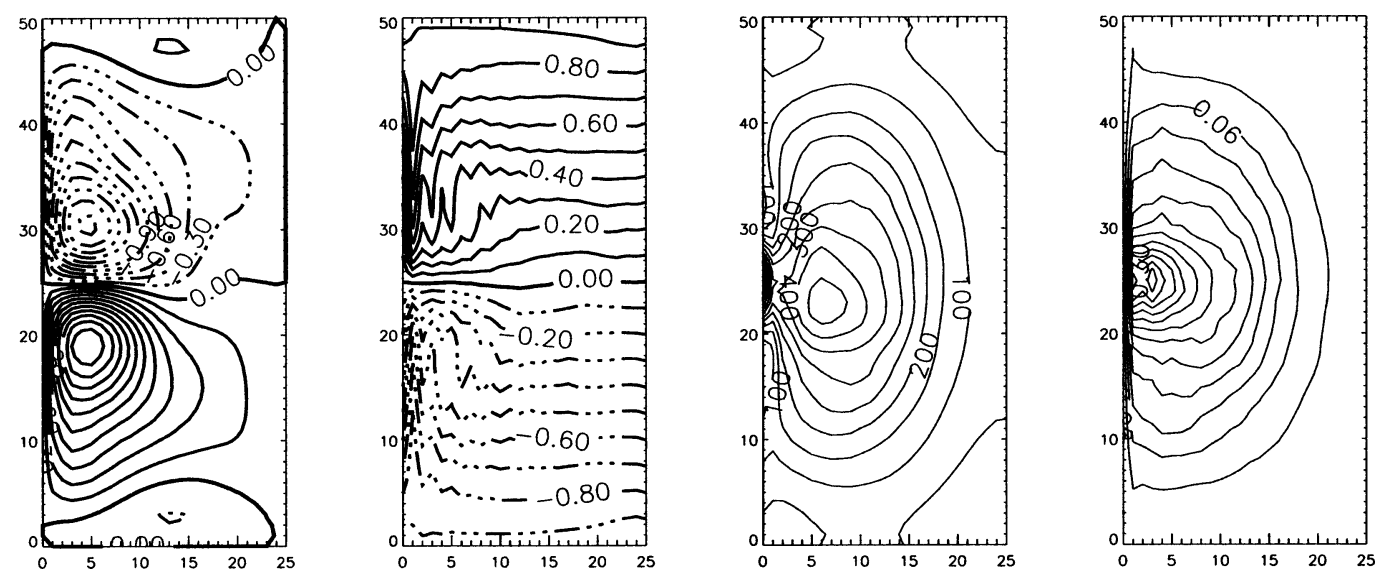

FIG. 3. When the simulation of Fig. 1 is repeated at a resolution 4 times as coarse-a grid resolution of $0.04 \mathrm{~L}-$ with no modeling of the subgrid scales, we find 1) the eddy flux of PV is so reduced that the outer pair of gyres is greatly weakened in comparison with Fig. 1, and 2) the insufficient resolution of the Munk layer scale $(0.02 L)$ results in grid-scale oscillations in the time-averaged PV field.

The traditional strategy in evaluating parameterizations or subgrid-scale models has been to compare LES models at coarse resolution with simulations which resolve the eddies and all relevant physical scales represented in the governing equations. Figure 4 shows the time-mean circulation resulting in the $\mathrm{BV}-\alpha$ model at a resolution 4 times as coarse. We remind the reader that the setting is identical to that of the previous case and the filter scale $\alpha$ has been set to the grid spacing $\Delta x$ which is now $0.04 \mathrm{~L}$. The following two improvements in the time-mean circulation with respect to Fig. 3 are observed: First, the outer eddy-driven gyres reappear as in the high-resolution run (Fig. 1). Second, the western boundary current region has been smoothed to be resolvable with the coarse resolution being used. In doing so, the grid-scale oscillations in the time-averaged potential-vorticity field in Fig. 3 have been largely eliminated. Note that the filter scale alpha (which is a priori specified to be the grid scale) exceeds the Munk layer scale. Hence, in obtaining the outer eddy-driven gyres, proper resolution of the Munk layer is not as much of an issue with the $\mathrm{BV}-\alpha$ model as it is with the original $\mathrm{BV}$ equation. This is an added advantage of the BV- $\alpha$ model.

On the other hand, in comparison to the high-resolution run, the BV- $\alpha$ model run seems to be too energetic (or insufficiently dissipative). This is indicated by the elevated levels of both mean and eddy kinetic energies. (Compare the first panels and the third panels in Figs. 1 and 4.) This enhanced energy is consistent with 1) the enhanced inverse cascade of energy due to the alpha regularization for 2D flows that was observed and explained in terms of scaling arguments in Nadiga and Shkoller (2001) and 2) the qualitative nature of alpha models to enhance transport over dissipation.

When the 4-times-as-coarse simulation at higher vis-
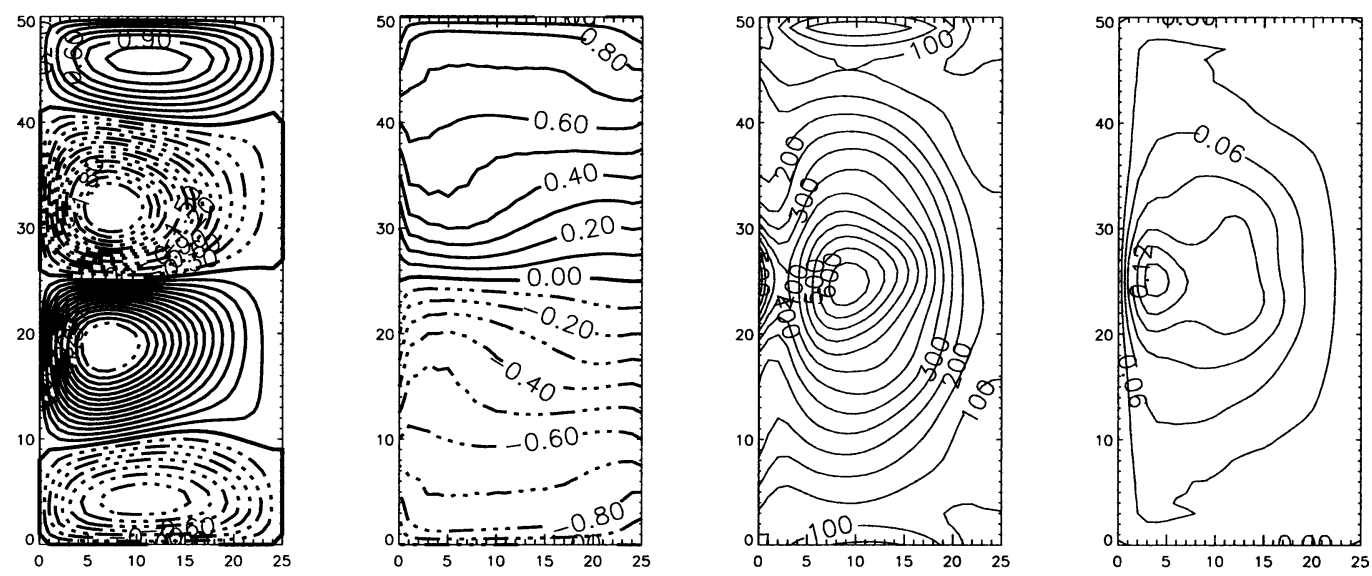

Fig. 4. When the 4-times-as-coarse simulation of Fig. 3 is repeated with the alpha model to account for subgrid scale activity, 1) the outer pair of gyres is restored and 2) the grid-scale oscillations in the PV field are greatly reduced. However, the strength of both the wind-driven and the eddy-driven mean circulation is too high in comparison with the resolved simulation of Fig. 1. 

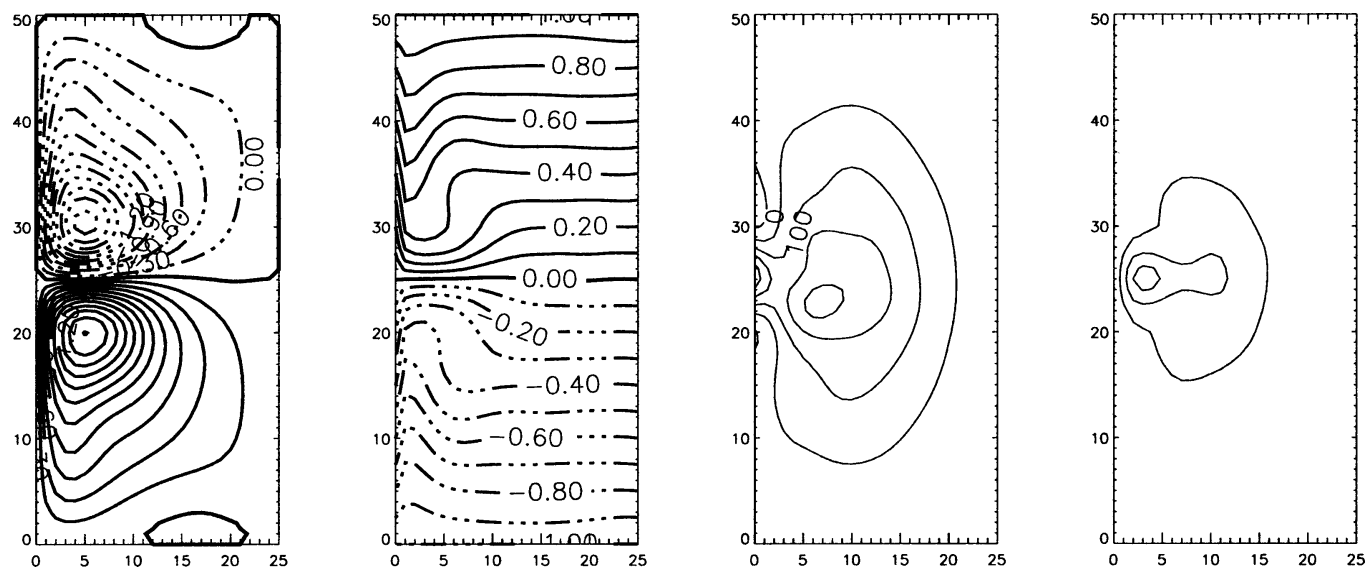

FIG. 5. When the 4-times-as-coarse simulation of Fig. 2 is repeated with the alpha model, the modification to Fig. 2 is minimal, verifying that the BV- $\alpha$ model does not produce spurious outer gyres in parameter regimes where they do not exist at high resolution.

cosity shown in Fig. 2 is repeated with the alpha model (Fig. 5), the modification to Fig. 2 is minimal. This verifies that the BV- $\alpha$ model does not produce spurious outer gyres in parameter regimes where they do not exist in a high-resolution run.

Figure 6 shows the time-mean circulation resulting in the BV-Leray- $\alpha$ model at the 4-times-as-coarse resolution, with identical settings as in the previous case. Again, there are clear improvements over the results of Fig. 3, the case with no modeling. However, while the improvements are qualitatively similar to those obtained using the previously discussed $\mathrm{BV}-\alpha$ model, quantitatively they are inferior to those shown by the BV- $\alpha$ model. Furthermore, at 8-times-as-coarse resolution, while the BV- $\alpha$ model continues to correctly model the high-resolution run (Fig. 7), the dissipation is too low for the BV-Leray- $\alpha$ model to run stably. The unstable behavior of BV-Leray- $\alpha$ model arising at a resolution 8 times as coarse is similar to that of the BV Clarkalpha model arising at the 4-times-as-coarse resolution.
While we do not present results from yet-coarser runs of the BV- $\alpha$ model, it is clear that the BV- $\alpha$ model will begin to fail when the grid becomes so coarse as to be unable to resolve the (turbulent) width of the WBC in Fig. 1 (about 0.25L).

\section{Discussion}

Unfortunately, studies of the alpha model in 2D are presently limited to a handful in number. Nadiga (2000) considered scaling behavior of the 2D Euler-alpha model in a doubly periodic, inviscid, and unforced setting and demonstrated how equipartition of energy and enstrophy leads to preservation of spectral scalings at large scales but allows the energy to fall off more rapidly at small scales. Nadiga and Shkoller (2001) considered forced-dissipative studies of energy and enstrophy cascades and noted besides the steeper falloff of energy at small scales, the amplification of the inverse cascade of energy. They also looked into resolution requirements
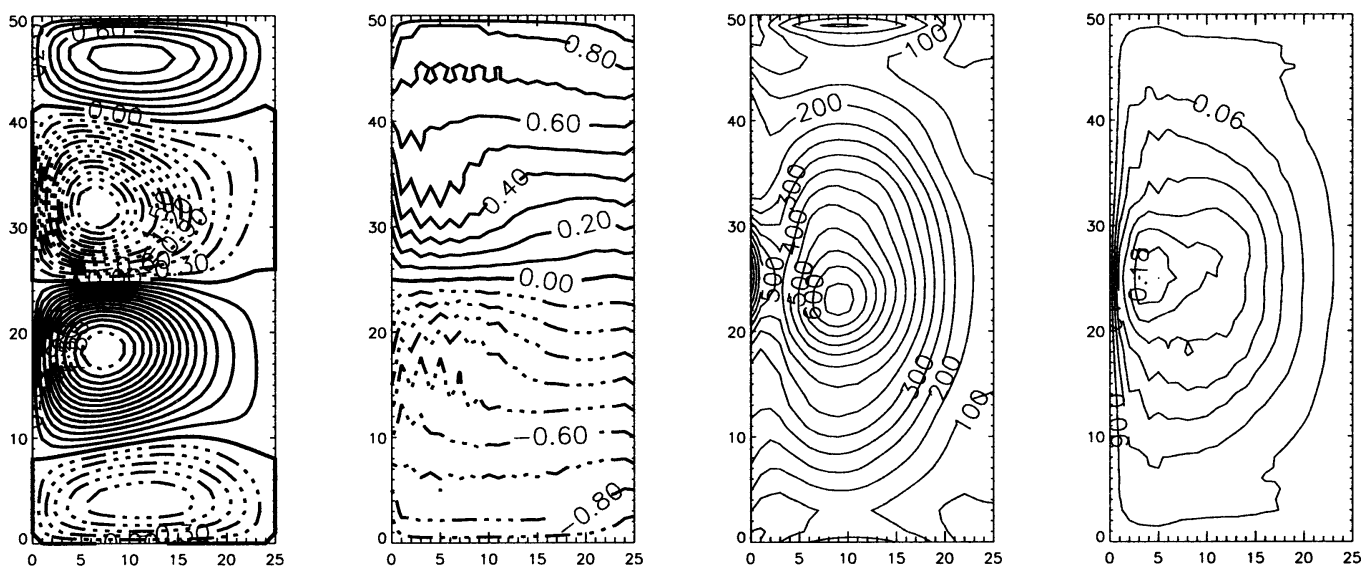

FIG. 6. When the Leray- $\alpha$ model is used instead of the full alpha model in the run of Fig. 4, the improvements with respect to Fig. 3 are qualitatively the same as with the alpha model, but they are quantitatively inferior. 

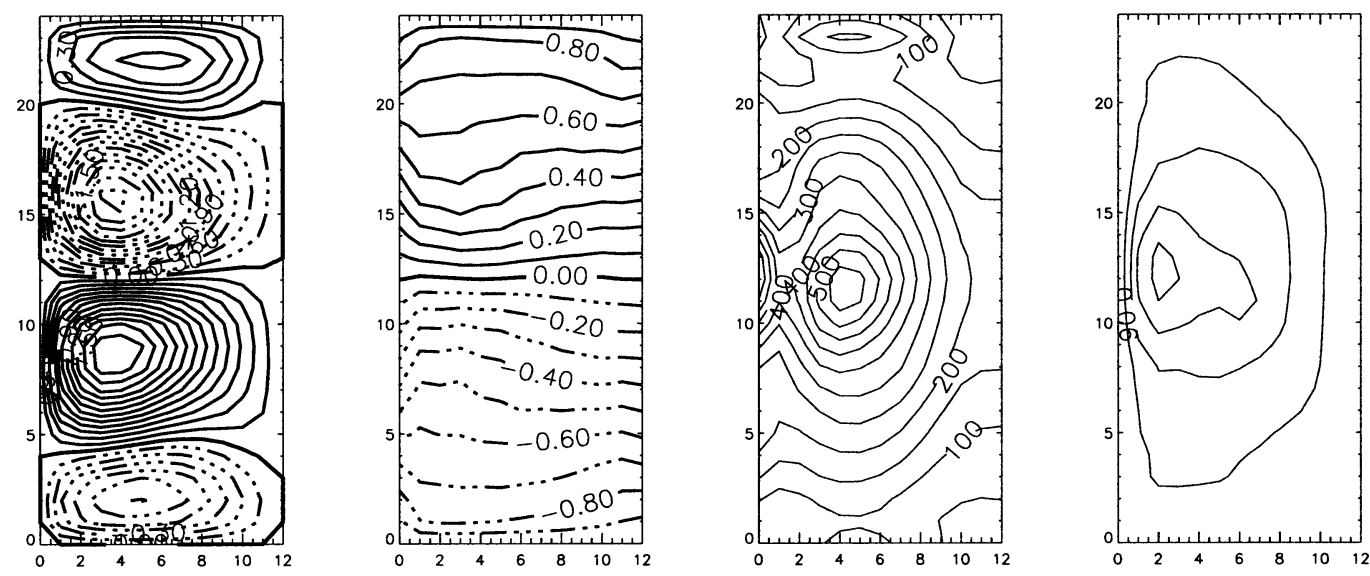

FIG. 7. At a resolution 8 times as coarse, only the alpha model continues to model the mean circulation, the eddy kinetic energy, and the eddy enstrophy reasonably well.

in that article. Nadiga and Margolin (2001) took the point of view of Reynolds averaging in the context of the double-gyre circulation in a closed ocean basin, showed how the steady-state circulation of the alpha model recovered the time-mean four-gyre circulation for appropriately chosen alpha and dissipation parameters, and discussed aspects of vorticity balance. That method, however, suffered from the problem of having to choose two parameters, based on the eddy-resolving run, and that notwithstanding the correspondence between the time mean of the eddy-resolving run and the alphaparameterized run was not very good. In the present study, by fixing a priori all parameters and changing only the resolution and taking a large-eddy-simulation point of view, we not only improve on the previous representation of the time-mean circulation, but also do a reasonable job of representing eddy kinetic energy and eddy potential enstrophy.

At the 4-times-as-coarse resolution, the three main cases (the BV equation with no model, the BV- $\alpha$ model and the BV-Leray- $\alpha$ model) all run stably in the doublegyre problem being considered. However, the Clark and filtered Clark LES models do not run stably and require additional dissipation to stabilize them. At the 8-timesas-coarse resolution, the BV-Leray- $\alpha$ model seems to fail in a manner similar to that in which the Clark model fails at the 4-times-as-coarse resolution, while the BV$\alpha$ model continues to run stably.

From a numerical standpoint, the explanation for why the BV and BV- $\alpha$ models run stably (at any resolution), while the other models do not, is because the Arakawa form of the Jacobian ensures nonlinear stability of the discretization, provided the equations can be written in a potential-vorticity advection form. While the $\mathrm{BV}$ and $\mathrm{BV}-\alpha$ equations have this property, the other models do not.

However, notwithstanding the numerical stability properties of the BV equation with no model at the 4and 8-times-as-coarse resolutions, these simulations at coarse resolution are seriously deficient when compared with the eddy-resolving simulations. At these coarse resolutions, the $\mathrm{BV}$ equation with no model is unable to capture the time-mean four-gyre circulation and is also unable to resolve the Munk layer. The latter at coarse resolutions generates grid-scale oscillations in the time-mean potential-vorticity field. While the BV$\alpha$ model successfully overcomes both of these deficiencies at either of the coarse resolutions, the BV-Leray- $\alpha$ model is less successful and then only at the 4-timesas-coarse resolution. On the other hand, the enhanced inverse cascade of energy in the BV- $\alpha$ model (Nadiga and Shkoller 2001) produces mean and eddy kinetic energies that are too high in comparison with the eddyresolving run, although they are still reasonable in the cases studied here.

From the point of view of geophysical fluid dynamics, the BV- $\alpha$ model shows that the tendency of eddies to mix potential vorticity in certain situations can be successfully parameterized in a manner very different from the usual dissipative mixing term that arises from assuming a downgradient form for the eddy flux of BV. The BV- $\alpha$ model is also significantly better than the usual downgradient mixing parameterization in its preservation (or even realistic enhancement) of variability.

From the downgradient parameterization investigations in Greatbatch and Nadiga (2000), eddy mixing of potential vorticity seems to be main driving mechanism for the outer set of gyres, and in the eddy-resolving simulations this process is manifestly due to nonlinear interactions (rather than weakly interacting basin-scale Rossby waves.) It then seems, in short, to be the capability of the nonlinear dispersive modification in the $\mathrm{BV}-\alpha$ model of the nonlinearity in the $\mathrm{BV}$ equation to (a) mix potential vorticity (Nadiga and Margolin 2001) and (b) deemphasize the importance of small scales (Nadiga 2000; Nadiga and Shkoller 2001) that makes the model work.

As to why the alpha model can accomplish this, consider the Rossby wave dispersion relation for the original flow 


$$
\omega=U k-\frac{\beta k}{|\mathbf{k}|^{2}},
$$

where $U$ is the ambient zonal flow, $|\mathbf{k}|^{2}=k^{2}+l^{2}, k$ is the wavenumber in the east-west direction and $l$ is the wavenumber in the north-south direction. One can see that short Rossby waves are frozen into the flow, in the sense that the phase velocity goes to zero as $|\mathbf{k}|^{-2}$, whereas long Rossby waves propagate through the flow. The alpha model exploits the above frozen nature of the small scales to provide a computational subgrid-scale model for the effects of the unresolved scales (that are smaller than $\alpha$ ) on the resolved flow. Note that, in the alpha model, as in the original flow, eddies and Rossby waves that are large in comparison with alpha propagate through the flow. This can be seen from the dispersion relation for the BV- $\alpha$ model (15). Furthermore, the phase speed of unresolved short Rossby waves goes to zero faster $\left(\propto|\mathbf{k}|^{-4}\right)$ than in the original equation. Thus, consistent with the large-eddy-simulation point of view that we adopt, the larger of the eddies that are generated by the meandering eastward free jet are being resolved while the smaller ones are being implicitly modeled by the alpha modifications to the original equations.

Modeling the effects of unresolved scales on the resolved large-scale circulation is a topic of ongoing research in geophysical fluid dynamics. The LANS- $\alpha$ subgrid-scale model/regularization that we used here in the context of the BV equation is a promising and new LES approach for parameterizing subgrid-scale eddy activity. Not only does this model retain useful mathematical structure (e.g., Kelvin circulation theorem) but also, as the present investigations suggest, shows accuracy and robustness in sustaining the four-gyre pattern in the time-mean circulation found in eddy-resolving simulations under the classic double-gyre forcing. The computational overhead associated with the alpha models was considerably less than that required for dynamic LES models, and no introduction of ad hoc parameters was required.

In future work, we will investigate the use of these alpha models in applications using multilayer quasigeostrophic and 3D primitive equations. Further, noting that in the quasigeostrophic approximation the computational overhead associated with these models is insignificant in comparison with the gains made by decreased resolution, we envisage being able to make these models similarly inexpensive in the context of primitive equations as well by using local filtering in place of the nonlocal Helmholtz operator inversion $H_{\alpha}^{-1}$, following the procedure of Geurts and Holm (2002).

Acknowledgments. We are grateful to L. G. Margolin, R. J. Greatbatch, C. Foias, J. E. Marsden, S. Shkoller, V. Putkaradze, and E. Titi for many stimulating discussions of the boundary conditions and other attributes of the LANS- $\alpha$ model. We thank two anonymous referees for their constructive criticism of earlier versions of the manuscript, which led to significant improvements in the presentation of the results.

\section{REFERENCES}

Chen, S., C. Foias, D. D. Holm, E. Olson, E. S. Titi, and S. Wynne, 1998: The Camassa-Holm equations as a closure model for turbulent channel flow. Phys. Rev. Lett., 81, 5338-5341.

— - D. D. Holm, L. G. Margolin, and R. Zhang, 1999: Direct numerical simulations of the Navier-Stokes alpha model. Physica $D, 133,66-83$.

Clark, R. A., J. H. Ferziger, and W. C. Reynolds, 1979: Evaluation of subgrid-scale models using an accurately simulated turbulent flow. J. Fluid Mech., 91, 1-16.

Foias, C., D. D. Holm, and E. S. Titi, 2001: The Navier-Stokes-alpha model of fluid turbulence. Physica D, 152, 505-519. [Available online from http://xxx.lanl.gov/abs/nlin.CD/0103037.]

,$- \ldots$, and -2002 : The three dimensional viscous CamassaHolm equations, and their relation to the Navier-Stokes equations and turbulence theory. J. Dyn. Differ. Equations, 14, 135. [Available online from http://xxx.lanl.gov/abs/nlin.CD/ 0103039.]

Geurts, B. J., and D. D. Holm, 2002: Alpha-modeling strategy for LES of turbulent mixing. Turbulent Flow Computation, D. Drikakis and B. G. Geurts, Eds., Kluwer, 237-278.

Greatbatch, R. J., and B. T. Nadiga, 2000: Four gyre circulation in a barotropic model with double gyre wind forcing. J. Phys. Oceanogr., 30, 1461-1471.

Holland, W. R., and L. G. Lin, 1975: On the generation of mesoscale eddies and their contribution to the ocean general circulation, I. A preliminary numerical experiment. J. Phys. Oceanogr., 5, 642-657.

_ , and P. B. Rhines, 1980: An example of eddy-induced ocean circulation. J. Phys. Oceanogr., 10, 1010-1031.

Holloway, G., 1986: Eddies, waves, circulation, and mixing: Statistical geofluid mechanics. Annu. Rev. Fluid Mech., 18, 91-147.

Holm, D. D., 1999: Fluctuation effects on 3D Lagrangian mean and Eulerian mean fluid motion. Physica $D, \mathbf{1 3 3}, 215-269$.

Leonard, A., 1973: Energy cascade in large-eddy simulations of turbulent fluid flows. Advances in Geophysics, Vol. 18, Academic Press, 237-248.

Leray, J., 1934: Sur les movements d'un fluide visqueux remplaissant l'espace. Acta Mathematica, 63, 193-248.

Marsden, J. E., and S. Shkoller, 2001: Global well-posedness for the Lagrangian averaged Navier-Stokes (LANS-alpha) equations on bounded domains. Philos. Trans. Roy. Soc. London, 359A, $1449-1468$

Nadiga, B. T., 2000: Scaling properties of an inviscid mean-motion fluid model. J. Stat. Phys., 98, 935-948.

_- and L. G. Margolin, 2001: Dispersive-dissipative eddy parameterization in a barotropic model. J. Phys. Oceanogr., 31, 25252531.

_ _ and S. Shkoller, 2001: Enhancement of the inverse-cascade of energy in the two-dimensional averaged Euler equations. Phys. Fluids, 13, 1528-1531.

Peterson, K. A., and R. J. Greatbatch, 2001: Vorticity fluxes in shallow water ocean models. Atmos.-Ocean, 39, 1-14.

Sadourny, R., and C. Basdevant, 1985: Parameterization of subgrid scale barotropic and baroclinic eddies in quasigeostrophic models: Anticipated potential vorticity method. J. Atmos. Sci., 42, $1353-1363$

Taylor, G. I., 1938: The spectrum of turbulence. Proc. Roy. Soc. London, 164A, 476-490.

Winckelmans, G. S., A. A. Wray, O. V. Vasilyev, and H. Jeanmart, 2001: Explicit-filtering large-eddy simulation using the tensordiffusivity model supplemented by a dynamic Smagorinsky term. Phys. Fluids, 13, 1385-1403. 